\title{
Synthesis of Novel Chlorhexidine Spheres with controlled release from a UDMA-HEMA Resin using Ultrasound.
}

Dong Luo ${ }^{\mathrm{a}}$, Saroash Shahid ${ }^{\mathrm{b}}$, Gleb B. Sukhorukov ${ }^{\mathrm{a}}$, Michael J. Cattell ${ }^{\mathrm{b} *}$

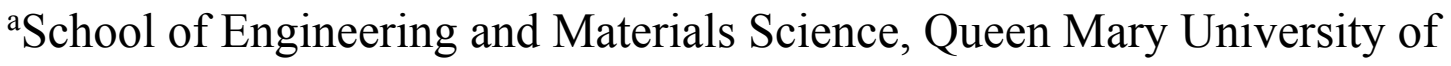

London, London, Mile End Road, E1 4NS, UK. ' $\mathrm{C}$ entre for Adult Oral Health, Bart's and the London, School of Medicine and Dentistry, Queen Mary University of London, Turner Street, Whitechapel, E1 2AD, UK.

*corresponding author; email address: $\underline{\text { m.cattell@qmul.ac.uk }}$ Telephone: 0044-0207 8826316 


\section{Abstract}

Objectives: Establish the release kinetics of new chlorhexidine particles incorporated in a dental resin, and with the application of ultrasound.

Methods: Spherical chlorhexidine particles (SCP) were synthesized (5 wt. \%), freeze dried and incorporated into UDMA-HEMA resins. Chlorhexidine diacetate (CDP) (5 wt. \%) was similarly incorporated in separate resins. Resin discs were immersed in deionized water, and a release profile established $(650 \mathrm{~h})$. Ultrasound was used to trigger chlorhexidine (CHX) release from the resin discs at specific durations (10-30s) and time intervals (1 to $425 \mathrm{~h}$ ). Chlorhexidine content was determined by UV-Vis absorption. The chlorhexidine particles/polymer composites were characterized using TGA, SEM, and confocal microscopy.

Results: SCP exhibited structures with high chlorhexidine content (90-95\%), and a Mean (SD) diameter of $17.2(2.5) \mu \mathrm{m}$ which was significantly $(\mathrm{p}<0.001)$ smaller than the CDP crystals at 53.6 (33.7) $\mu \mathrm{m}$. The SCP discs had a lower (7.7\%) CHX release compared to the CDP group (16.2\%). Ultrasonication of the resin discs with increasing durations $(10-30 \mathrm{~s})$ resulted in higher drug release rates. CDP release rates (CHX) over $650 \mathrm{~h}$ were: $23.5 \%(10 \mathrm{~s}), 42.6 \%(20 \mathrm{~s}), 51.2 \%$ (30s), and for SCP (CHX) were; 9.8\% (10s), 12.3\% (20s), and 14.0\% (30s). SEM/confocal microscopy revealed CDP discs exhibited dissolution associated with the particle surface and SCP from the interior.

Significance: Chlorhexidine spheres incorporated in a dental resin demonstrated a responsive and lower CHX release. Ultrasound enhanced CHX release and is useful in clinical situations where the drug is required on demand to treat severe or persistent infections.

Key Words: Chlorhexidine, Ultrasound, Dental resins, Drug delivery. 


\section{Introduction}

Since being introduced to dentistry in the 1950s, dental composites have undergone considerable changes such as; the modification of fillers to enhance the mechanical properties and the optimisation of the polymeric matrix to improve their biocompatibility [1]. Currently available dental composites have exceptional aesthetics and comparable mechanical properties (flexural strength, fracture toughness and tensile strength) when compared with porcelain and amalgam, and superior to that of glass ionomers [2]. This allows their wide use for anterior and posterior tooth restorations, as well as pit and fissure sealants [3]. Dental composites are cured by light and chemically initiated free radical polymerization which results in 2-5.63 vol. \% shrinkage of the material [4]. This can lead to the formation of gaps between the restoration and the tooth [5]. Bacterial microleakage through these gaps can cause marginal discoloration, sensitivity, inflammation, recurrent caries and apical periodontitis [6]. Furthermore, accumulation of bacterial biofilms on the surface of dental composites can lead to degradation of the polymer matrix, resulting in the weakening of the restoration. Dental composites with antimicrobial properties are therefore highly desirable to overcome these problems.

Chlorhexidine is a bis-biguanide antiseptic and disinfectant that has a bactericidal and bacteriostatic action against a wide range of gram-positive and gram-negative bacteria $[7,8]$, and has been incorporated in a variety of resin composites [9-11]. Chlorhexidine also stabilizes the resin-dentine bond by inhibiting matrix metalloproteinases (MMP) which are responsible for the breakdown of the bond at the dentine-restoration interface [12]. Inclusion of chlorhexidine into dental composites has been achieved by mixing the chlorhexidine diacetate with monomers [13-15]. This composite however suffers from an uncontrollable release of chlorhexidine, due to its rapid diffusion from the methacrylate based resin. To overcome these drawbacks efforts have been made to develop new chlorhexidine formulations [16-19] and investigate novel drug carriers [20,21], to control chlorhexidine release. Ultrasound is another 
method previously used to control the release of antibacterial agents in bone cements, where the synergistic effect of ultrasound and antibiotics enhanced antimicrobial efficiency [15, 22]. More recent studies utilised high frequency ultrasound to deliver antimicrobial chitosan nanoparticles into dentinal tubules, which may open up new approaches for the treatment of root canal infections $[23,24]$.

Recently Luo et al. [25] developed a novel chlorhexidine compound, with unique spherical morphology and the ability to provide controlled release of chlorhexidine. The aim of this study was therefore to investigate the effects of ultrasound from a dental scalar (ultrasound probe); to enhance drug release from novel chlorhexidine spheres in a light cured dental resin. Ultrasound triggered chlorhexidine release may be particularly useful in developing triggered drug delivery from a polymer system incorporating novel chlorhexidine spheres. 


\section{Materials and Methods}

\subsection{Fabrication of spherical chlorhexidine particles}

Chlorhexidine spheres were fabricated and characterized as described previously [25]. Briefly, $15 \mathrm{mg} / \mathrm{ml}$ chlorhexidine diacetate (Sigma-Aldrich, Lot: $19 \mathrm{H} 0417)$ solution and $0.33 \mathrm{M} \mathrm{CaCl}_{2}$ (Sigma-Aldrich, Lot: SLBF7416 V) solution were mixed at 1:1 ratio by volume at room temperature. The mixture was shaken for $1 \mathrm{~min}$, and then centrifuged at $2000 \mathrm{rpm}$ for $1 \mathrm{~min}$ (Eppendorf centrifuge 5417C, Germany). The sedimentation was then washed three times with $0.33 \mathrm{M} \mathrm{CaCl}_{2}$ solution and then centrifuged again. Thereafter, the product was freeze dried (ScanVac Cool Safe Freeze Drying, Denmark) at $-107{ }^{\circ} \mathrm{C}, 0.009 \mathrm{mBar}$ for 1 day, and was used for all the following experiments. Several reproducible batches (3) were produced for this work.

\subsection{UV/VIS Spectroscopy and TGA Analysis}

The content of chlorhexidine in the spherical particles was determined by UV-Vis absorption (Lambda 35, Perkin Elmer, USA). Initially, a series of chlorhexidine diacetate aqueous solutions with standard concentrations of $0.25,1,2,3,4,5,10,20$ ppm were prepared, and the absorption measured. The absorption peak at $254 \mathrm{~nm}$ and the reference concentration had a linear relationship, and a standard curve of absorption verses concentration was established (Figure S1). Residual chlorhexidine in all the supernatants was determined by measuring the UV absorption of the supernatants at $254 \mathrm{~nm}$, and then calculating the concentration using the calibration curve. The proportion of chlorhexidine in the spherical particles was then calculated by subtraction (initial CHX $-\mathrm{CHX}$ in all supernatants $=\mathrm{CHX}$ in the particles). In addition, the freeze dried chlorhexidine spheres; chlorhexidine diacetate and $\mathrm{CaCl}_{2}$ powder were analyzed using Thermo-gravimetric analysis (TGA Q50, USA). The TGA was carried out at $10^{\circ} \mathrm{C} / \mathrm{min}$ in a nitrogen atmosphere, over a temperature range of $50-800^{\circ} \mathrm{C}$. 


\subsection{Preparation of chlorhexidine UDMA- HEMA resin discs}

The resin was prepared by mixing 64\% urethane dimethacrylate (UDMA) (Esschem, UK, Lot: 591-22), 36\% hydroxyethyl methacrylate (HEMA) (Aldrich, UK), 0.08\% of N, N-dimethyl-Ptoluidine (Acros Organics, UK) and 0.05\% dimethylamino ethyl methacrylate (Aldrich, UK). The mixture was stirred at $800 \mathrm{rpm}$ for $15 \mathrm{~min}$ (VWR Stirrer, USA). Finally, camphorquinone (Aldrich, UK) was added at the proportion of $0.1 \%$ and the mixture was stirred for another 15 mins. The current resin was selected as it was biocompatible when exposed to L929 fibroblasts (ISO 10993) and following a 28 day resin leach out study and DNA assay. Freeze dried spherical chlorhexidine particles were weighed (Salter Ander-180A weighing scale, UK) and incorporated within the resin at $5 \mathrm{wt} \%$ (chlorhexidine content), then placed in an Eppendorf tube and mixed for $15 \mathrm{~s}$ in a rotomix mixer $(120 \mathrm{~V} / 60 \mathrm{~Hz}, 2850$ rotations $/ \mathrm{min})(\mathrm{ESPE}$ RotoMix, USA). A separate set of samples containing chlorhexidine diacetate powder (5 wt $\%$ chlorhexidine content) was also incorporated in the prepared resin in the same way. The resin mixture was then placed into a Teflon mold (10 $\mathrm{mm}$ diameter $\times 2 \mathrm{~mm}$ depth) and cured through a Mylar film strip using a curing light (Bluedent LED pen, Bulgaria) (430-490 nm, 600 $\mathrm{mW} / \mathrm{sq} . \mathrm{cm}$ ) for $30 \mathrm{~s}$ on both sides. The discs were next weighed on a microbalance (Salter Ander-180A weighing scale, UK), and the chlorhexidine content in each disc was calculated. 


\subsection{Labelled chlorhexidine particles and confocal microscopy}

For visualization of the chlorhexidine sphere distribution in the resin composites, the spheres were labelled using rhodamine B (Sigma, Lot: 063K3407). Spherical chlorhexidine particles were synthesized as described in section 2.1; however before mixing the chlorhexidine diacetate solution with the $\mathrm{CaCl}_{2}$ solution, $100 \mu \mathrm{l}$ of rhodamine $\mathrm{B}(1 \mathrm{mg} / \mathrm{ml})$ was added. The synthesized particles were then centrifuged, washed and freeze dried as in section 2.1. The labelled spherical chlorhexidine particles ( $5 \mathrm{wt} \%$ chlorhexidine content) were mixed with UDMA-HEMA resin and placed into a mold (10 mm length, $10 \mathrm{~mm}$ width and $1 \mathrm{~mm}$ depth) and cured using a curing light as in section 2.3. The rhodamine $\mathrm{B}(\mathrm{RhB})$ labelled chlorhexidine particle resin specimen before and after immersion in water $(650 \mathrm{~h})$ was then characterized using confocal microscopy (Leica TS confocal scanning system, Germany).

\subsection{Chlorhexidine release kinetics}

In the release study, all UDMA-HEMA resin discs containing spherical chlorhexidine particles (SCP) or chlorhexidine diacetate powder (CDP) were stored in cuvettes containing $2 \mathrm{ml}$ deionized water at room temperature. To measure the effect of ultrasound on the release kinetics specimens were split into 4 groups ( $n=3$ per group) for each composite (SCP and CDP). The specimen groups comprised; Gp1:0s, Gp2:10s, Gp3:20s and Gp4:30s ultrasound exposure. Specimens were treated with a single application of ultrasound at multiple time points. The ultrasound was delivered by contacting the disc specimen surface with an ultrasound probe (27-30 kHz, Piezon Master 400, Electro Medical Systems, Switzerland) at the following time points; 1h, 3h, 5h, 15h, 25h, 40h, 65h, 95h, 140h and 205h. Ultrasound was applied to the all the samples identically, with the ultrasound probe in contact with the surface of resin discs and moved around the sample during application. Following the last ultrasonic treatment $(205 \mathrm{~h})$, release measurements were continued until $650 \mathrm{~h}(275 \mathrm{~h}, 350 \mathrm{~h}, 425 \mathrm{~h}, 500 \mathrm{~h}, 575 \mathrm{~h}$ and $650 \mathrm{~h})$. Solutions from each time intervals were collected for the UV-Vis absorption tests (Lambda 35, 
Perkin Elmer, USA) and replaced with fresh deionized water. The amount of released chlorhexidine was determined according to the established relationship of absorption and chlorhexidine concentration, and cumulative release curves were plotted. The rhodamine B labelled chlorhexidine particle resin specimen was also immersed in water for $650 \mathrm{~h}$ and fresh water added at each time interval.

To further investigate the effect of ultrasound on triggering chlorhexidine release, another set of experiments with the same time intervals and release measurements was conducted on the two composite materials. The first group 1 received no ultrasound treatment. Ultrasound (30s) was next delivered to the disc specimens at the start of the experiment (0h, Gp.2); at the start and at $205 \mathrm{~h}\left(14.3 \mathrm{~h}^{1 / 2}\right)(\mathrm{Gp} .3)$ and at the start, 205h $\left(14.3 \mathrm{~h}^{1 / 2}\right)$ and $425 \mathrm{~h}\left(20.6 \mathrm{~h}^{1 / 2}\right)(\mathrm{Gp} .4)$. The release profile of each group with different treatment times for each material was compared. The slope of release curves after each of ultrasound treatment was determined by $\Delta y / \Delta x$ and compared with that of the control.

\subsection{Scanning electron microscopy}

Chlorhexidine diacetate powder (C6143, Lot: 19H0417, CDP) and spherical chlorhexidine particle (SCP) powder samples were prepared by placing powder suspensions onto the surface of glass slides, which were stuck onto SEM stubs, and then dried in air. Resin discs containing CPD or SCP from each group before and after the ultrasonication study (sections 2.3, 2.5), were dipped into liquid nitrogen and broken. Cross-sections were mounted onto SEM stubs. All the samples were gold coated for 45s at $18 \mathrm{~mA}, 0.04 \mathrm{mBar}$ using a sputter coater (SC7620, Emitech, UK). All samples were characterized using a scanning electron microscope (FEI Inspect-F, USA), in the secondary electron imaging mode. An accelerating voltage of $10 \mathrm{kv}$, spot size of 3.5 and working distance of $10 \mathrm{~mm}$ was used. Multiple images (10) were collected for each of 
the samples. The Mean (SD) particle diameter and size range of the chlorhexidine spheres and chlorhexidine diacetate particles was measured using quantitative image analysis (Sigma Scan Pro 5, Systat Software Inc., UK) of the SEM images. Over 100 particles were measured and the data was compared using a t test (Sigma Stat, version 2.03, SPSS Inc., Chicago, IL, USA), to analyze statistically significant differences between groups $(\mathrm{P}<0.05)$.

\section{Results}

\subsection{Results of the SEM study}

The results of the SEM study show a wide particle distribution (6.4-194.7 $\mu \mathrm{m})$ of angular/tabular chlorhexidine diacetate crystals (Figures 1a, c), with a Mean (SD) diameter of $53.6(33.7) \mu \mathrm{m}$. The spherical chlorhexidine particles (Figures 1b, d) however, produced a narrower particle size range (6.3-21.7) and a Mean (SD) diameter of $17.2(2.5) \mu \mathrm{m}$. The particle size data was compared using a $\mathrm{t}$ test and the chlorhexidine particle group showed a significantly lower $(\mathrm{p}<0.001)$ Mean particle diameter than the chlorhexidine diacetate group. SEM photomicrographs of cross-sectional images of the UDMA-HEMA resin discs containing spherical chlorhexidine particles and chlorhexidine diacetate powder are shown in Figures 1e and 1f. The morphology of the novel spherical chlorhexidine particles allowed the resin to finely penetrate the interior structure of the particles (Figure 1f). This was in contrast to the resin interaction with the angular and irregular morphology of the tabular chlorhexidine diacetate crystals, which exhibited resin penetration on its external surfaces (Figure 1e). Cross sectional images of resin composite discs after ultrasonic treatments (10-30 s) indicated an increasing gap between the chlorhexidine diacetate crystals and the resin, with a Mean (SD) gap of $6.68(1.33) \mu \mathrm{m}(10 \mathrm{~s}$, Figure $4 \mathrm{c}), 10.74(0.87) \mu \mathrm{m}(20 \mathrm{~s})$ and $13.94(1.87) \mu \mathrm{m}(30 \mathrm{~s})$. Dissolution of the particle interior was in evidence in the spherical chlorhexidine particle resin specimens (Figure 4d, $10 \mathrm{~s}$ ultrasonics). 


\subsection{Results of the Thermo-gravimetric analysis}

The results of the TGA analysis are shown in Figure 2. After increasing the temperature to $800^{\circ} \mathrm{C}$ the $\mathrm{CaCl}_{2}$ powder had $74 \mathrm{wt} \%$, chlorhexidine diacetate had $4 \mathrm{wt} \%$ and the spherical chlorhexidine spheres had $8.5 \mathrm{wt} \%$ remaining. The decomposition temperature of the chlorhexidine spheres $\left(210^{\circ} \mathrm{C}\right)$ was also higher than the chlorhexidine diacetate crystals $\left(120^{\circ} \mathrm{C}\right)$.

\subsection{Release kinetics of chlorhexidine from resin discs}

The proportion of chlorhexidine in the spherical particles was determined to be $90-95 \mathrm{wt} \%$ according to the UV-Vis absorption results and calculating using the calibration curve (Figure S1). The release process of chlorhexidine from the spherical chlorhexidine particle filled resin showed a two stage release. The first stage is a slow release stage which lasted up to $205 \mathrm{~h}$ $\left(14.3 \mathrm{~h}^{1 / 2}\right)$, followed by a relatively rapid release which lasted till the end of the experiment at 650 h (Fig. 3b). Chlorhexidine diacetate showed a more single stage release in comparison, which lasted till 205 hours after which the release indicates it was starting to plateau (Fig. 3a). Without any ultrasonic's, chlorhexidine diacetate filled resin discs released $16.2 \%$ chlorhexidine (Fig. 3a), whereas the chlorhexidine sphere filled resin discs released 7.7\% chlorhexidine at $650 \mathrm{~h}$ (Fig. 3b). The effect of ultrasound on chlorhexidine release kinetics was significant, with longer sonication durations giving higher release rates. The cumulative release for chlorhexidine diacetate incorporated resin discs (650 h) was; $23.5 \%$ (10s), 42.6\% (20s), $51.2 \%(30 \mathrm{~s})$, and for chlorhexidine sphere resin discs was; $9.8 \%(10 \mathrm{~s}), 12.3 \%(20 \mathrm{~s})$, and $14.0 \%$ (30s) (Figures 3a, 3b).

To further demonstrate the sensitivity to ultrasound and to control the release kinetics of chlorhexidine from the UDMA-HEMA resin discs, ultrasound was also carried out at specific 
time intervals $\left(0 \mathrm{~h}, 14.3 \mathrm{~h}^{1 / 2}\right.$ and $\left.20.6 \mathrm{~h}^{1 / 2}\right)$. The chlorhexidine release kinetics of resin discs for each ultrasound treatment is illustrated in Figures $3 \mathrm{c}$ and $3 \mathrm{~d}$. For the chlorhexidine diacetate resin discs (Figure 3c), the influence of $30 \mathrm{~s}$ ultrasonication $(0 \mathrm{~h})$ indicated a moderate chlorhexidine burst release, and for the second ultrasonication treatment $\left(14.3 \mathrm{~h}^{1 / 2}\right)$, a more dramatic burst release, and the third ultasonication $\left(20.6 \mathrm{~h}^{1 / 2}\right)$ produced a final responsive release indicated by the change in slope (Table 1). In comparison, the chlorhexidine sphere resin discs were less sensitive to the ultrasonication after $30 \mathrm{~s}$ ultrasonication ( $0 \mathrm{~h}$, Figure $3 \mathrm{~d})$, and there was a delay until $150 \mathrm{~h}\left(12 \mathrm{~h}^{1 / 2}\right)$, where there was a small increased drug release. The second $\left(14.3 \mathrm{~h}^{1 / 2}\right)$ and third $\left(20.6 \mathrm{~h}^{1 / 2}\right)$ ultrasonication, however led to a change in slope (Table 1) and evidence of a responsive chlorhexidine release.

\subsection{Confocal microscopy results}

Confocal images of the labelled $(\mathrm{RhB})$ chlorhexidine spheres inside the UDMA-HEMA resin can be seen in Figures 4a, b. The dendritic structure is revealed at the red channel fully intact before the release experiment (Fig. 4a). Figure $4 \mathrm{~b}$ indicates the particle following the release experiment showing dark areas within the particle radiating from the central portion, but with the particle /resin interfaces still largely intact. These results indicated partial dissolution of the $\mathrm{RhB}$ associated with the particle center, and the presence of large amount of residual $\mathrm{RhB}$ still retained in the resin composite. 


\section{Discussion}

Due to its low solubility chlorhexidine is most commonly used in the form of chlorhexidine digluconate and chlorhexidine diacetate [7,8]. The problems concerned with applying chlorhexidine digluconate or incorporating chlorhexidine diacetate crystals into gels, wound dressings or resins for antimicrobial applications is their rapid release and the difficulty of achieving a sustained long term antibacterial effect [26]. A sustained drug release over a long time period is beneficial to effectively suppress bacterial growth and eliminate biofilms [27]. To achieve sustained release of chlorhexidine numerous carriers were studied, including microporous silica's [28] and polymeric micro and nanoparticles [29, 30], but low drug loading capability and the difficulty of controlling the release were problems. Complexing of chlorhexidine/ chlorhexidine digluconate with cyclodextrin and encapsulation using an emulsion-solvent evaporation technique and compression into chips also resulted in low drug loading efficiency $(9-16.8 \%)[31]$.

The approach in the current study was based on the co-precipitation of chlorhexidine diacetate with a $\mathrm{CaCl}_{2}$ to produce spherical chlorhexidine particles, with a controlled size range (6.321.7), dendritic morphology and large surface area (Fig 1b, 1d). This was in contrast to the chlorhexidine diacetate crystals with irregular and tabular morphology (Figs. 1a, 1c), with a significantly $(p<0.001)$ larger crystallite size and broader size range $(6.4-194.7 \mu \mathrm{m})$. The current synthesis process allowed the nucleation and growth of crystallites, where the $\mathrm{Cl}^{-}$ anions were thought to be associated with the precipitation of the crystallite and the cation coordinated with the structure of the chlorhexidine [25]. A high chlorhexidine content (90-95 $\mathrm{wt} \%$ ) as determined by UV-Vis absorption was also achieved. TGA confirmed the weight loss for the $\mathrm{CaCl}_{2} \cdot 2 \mathrm{H}_{2} \mathrm{O}$ was $26 \mathrm{wt}$ \%, which was thought to be due to the loss of $\mathrm{H}_{2} \mathrm{O}$ (theoretically is $24.5 \mathrm{wt} \%$ ). It was therefore assumed that the $\mathrm{CaCl}_{2}$ did not decompose (Figure 2). The remaining $4 \mathrm{wt} \%$ for chlorhexidine diacetate may be associated with degradation products, 
whilst the increased $4.5 \mathrm{wt} \%$ (remaining weight of spherical CHX particles - remaining weight of chlorhexidine diacetate/degradation products) was ascribed to the $\mathrm{CaCl}_{2}$ in the spherical particles. Chlorhexidine compounds have been synthesized by mixing chlorhexidine digluconate and sodium hexametaphosphate solutions together to produce antibacterial chlorhexidine hexametaphosphate nano-particles and aggregates for use in glass ionomers and biomedical applications $[17,18]$. Other chlorhexidine formulations based on the coordinating ability of biguandines with metal ions $\left(\mathrm{Cu}^{2+}, \mathrm{Zn}^{2+}, \mathrm{Sn}^{2+}, \mathrm{Ag}+\right)$, were also reported, but with no structure or morphology of the complexes revealed [19, 32, 33]. The current formulations produced a specific and reproducible crystallite size and morphology which did not suffer from aggregation behavior or any potential issues with nano-toxicity [34], which might affect nanomaterials without a carrier.

The release kinetics of the chlorhexidine diacetate and chlorhexidine spheres incorporated in the UDMA-HEMA resins were evaluated before the application of ultrasonics. With an equivalent amount of chlorhexidine ( $5 \mathrm{wt} \%$ ), the resin discs containing the chlorhexidine spheres had a lower chlorhexidine release (7.7\%), compared to that of chlorhexidine diacetate $(16.2 \%)$ after $650 \mathrm{~h}$ water storage (Figures $3 \mathrm{a}, 3 \mathrm{~b}$ ). It was also apparent from the change in slope (Table 1) that the chlorhexidine diacetate release data appeared to show signs of a plateau compared with the chlorhexidine sphere specimen data over $650 \mathrm{~h}$. It was assumed that higher solubility of chlorhexidine diacetate compared with the spherical chlorhexidine compounds was in part due to the presence of $\mathrm{Ca}^{2+}$ and $\mathrm{Cl}^{-}$in the structure and associated with the different release kinetics observed. Zeng et al. [7] commented that the quantity of chlorhexidine in solution was associated with the types of ion in solution, with lower concentrations when dichlorides were present due to structural solubility. It was thought that when the dihydrochloride salts KSP was lower than the diacetate salt, the formation of a low solubility 
chlorhexidine dihydrochloride was more thermodynamically possible [35]. The higher release data for chlorhexidine diacetate incorporated into resins has also been linked to higher levels of residual monomer, caused by inhibition of the polymerization process [36]. A correlation between rapid chlorhexidine release rates and water sorption of the polymer was reported, due to the presence of unreacted monomer and hydrophilic resin components [37]. The stabilization of free radicals by chlorhexidine diacetate addition may also slow the termination step in polymerization of HEMA, TEGDMA and UDMA resin blends [38]. The release mechanism was thought to be due to droplet formation around the chlorhexidine salt, and with drug diffusion dependent on such droplets linked to the surface via the formation of pathways or channels [39]. Another factor may be the increased surface area of spherical chlorhexidine particles allowing the polymer resin (UDMA-HEMA) to finely penetrate their interior structure (Figure 1f). SEM and confocal microscopy confirmed the efficacy of the resin penetration and RhB labelling suggests some preservation of the chlorhexidine within the polymer before water storage (Figure 4a). Rhodamine B dissolution from the particle interior following the release experiment (650 $\mathrm{h}$ water storage, no ultrasonics) might indicate that partial drug dissolution within the chlorhexidine sphere (Figure 4b) may have occurred. The interaction of the resin with the larger and angular morphology of the tabular chlorhexidine diacetate crystals was on its external surfaces (Figure 1e) which may have encouraged increased water sorption. Inagaki et al. [40] commented on the importance of the chemical composition of the monomer in resin blends containing chlorhexidine, their water sorption, polymerization and plasticization, with chlorhexidine dissolved with unreacted monomer causing spaces between the polymer bulk.

The release profile of chlorhexidine diacetate from the resin composite was a complicated process, with dissolution of the drug by a burst release from the surface initially, followed by a sustained diffusion controlled release (Figure 3a) and similar to other studies [37, 39]. 
Mechanisms for burst released were reviewed by Haung and Brazel [41], and one reason was drug entrapment on or near the surface and instantaneous leaching in water, and a lack of physiological or chemical binding to the polymer network [22]. Subsequent diffusion of water into the resin matrix and drug dissolution leads to porosity, allowing further drug diffusion at interconnected pores, and affecting release rates at higher drug loading [42]. According to the release profile in Figure 3a (0s group), most of the incorporated chlorhexidine diacetate remains trapped within the resin composite, with the release increased from $16.2-51.2 \%$ at 650 $\mathrm{h}$ using ultrasonics (0-30 s treatments). Triggers such as ultrasound are useful to release any remaining drug especially when the concentration of released antimicrobial agents is below the minimum inhibition concentration (MIC). This method has previously been reported to increase gentamicin and vancomycin release from acrylic bone cements [22], which resulted in enhanced in vivo bacterial inhibition and inflammation relief [15]. Chlorhexidine release was enhanced by ultrasound treatment for both the chlorhexidine diacetate and chlorhexidine sphere resin composites, with longer ultrasonication time (10 to 30s) enhancing chlorhexidine release (Figs. 3a, 3b). It is feasible that micro streaming induced by ultrasound was the main reason, and with cavitation in the low-frequency range $(18-100 \mathrm{kHz})$ [43], which is beneficial for fluid exchange and encouraged chlorhexidine to diffuse out [44]. An aqueous channel/pathway is required for chlorhexidine release from resin composites, so the influence of ultrasonication on this process may be dramatic. In particular, removal of residual monomer has been demonstrated in auto-polymerized PMMA at low ultrasonic frequency $(28 \mathrm{kHz})$ [45]. The thermal effects generated by ultrasonication should also be taken into consideration [46], since the influence of ultrasound on gentamicin release was equal to a $3^{\circ} \mathrm{C}$ increase in temperature [22]. Clinical applications in dentistry often report higher ultrasonication temperatures $\left(>10^{\circ} \mathrm{C}\right)$, but this is dependent on the equipment set up and application [47]. It can be assumed that heat generated during ultrasonication may have assisted the dissolution of chlorhexidine compounds 
in the resin composites. Increased water sorption, solubility and cracking in HEMA containing polymer blends is also reported at higher temperatures [48].

Ultrasonication of the resin samples containing chlorhexidine spheres exhibited lower level drug release (9.8-14\%), via a step wise process and showed no signs of a plateau at $650 \mathrm{~h}$. This behavior was also illustrated when ultrasonication was used at different time points (start, 14.3 $\mathrm{h}^{1 / 2}, 20.6 \mathrm{~h}^{1 / 2}$ ), but with a clear change in slope at the $14.3 \mathrm{~h}^{1 / 2}, 20.6 \mathrm{~h}^{1 / 2}$ showing a responsive drug release (Figure 3d, Table 1). Ultrasonics (30 s) at the start (0h) showed little change in slope (0-3 h) compared no ultrasonics (Table 1), but with a later release onset $\left(150 \mathrm{~h}, 12 \mathrm{t}^{1 / 2}\right)$. This may be related to the morphology, chemical composition and resin penetration of the drug spheres and the time taken to penetrate the polymer with water, as a more responsive release was noted for later ultrasonic treatments (Figures. 1b, 1d). This reservoir or storage effect until the structure is penetrated by water [41], influencing the release kinetics and retention rate of chlorhexidine. The chlorhexidine diacetate resin specimens subjected to $30 \mathrm{~s}$ ultrasonics at the start (0h) produced a higher drug release, with a more responsive burst release similarly evident once the polymer had been exposed to longer water storage by the $14.3 \mathrm{~h}^{1 / 2}$ treatment (Figure 3c). Water uptake studies of the unfilled resin in the current study indicated that equilibrium was reached within this time period but is dependent on the resin composition, hydrophilicity and crosslink density [49].

The particle size, surface area, morphology and chemistry of incorporated chlorhexidine compounds may have an influence on the release kinetics of the respective particles added. The larger size (Mean diameter (SD)) of 53.6 (33.7) and tabular morphology of the chlorhexidine diacetate crystals were more sensitive to the effects of ultrasound than the homogeneous and smaller spheres (Figures 1d, 1f). The morphology of the solid chlorhexidine diacetate particle and cleavage planes (Figures. 1c and 1c) may be more susceptible to solubility, 
and damage by collapse cavitation, high velocity ultrasonic shock waves and other processes [50], since more dramatic burst release was observed corresponding to each of the ultrasound treatment at $14.3 \mathrm{~h}^{1 / 2}, 20.6 \mathrm{~h}^{1 / 2}$ (Fig. 3c). Cross-sectional images of resin composite discs after ultrasonic treatments (10-30 s) indicated an increasing Mean (SD) gap width between the chlorhexidine diacetate crystals and the resin of $6.68(1.33) \mu \mathrm{m}(10 \mathrm{~s}$, Figure 4c) to 13.94 (1.33) $\mu \mathrm{m}(30 \mathrm{~s})$. It was therefore thought that this was largely a surface dissolution process, whereas the chlorhexidine sphere dissolution was associated with the sphere center (Figures 4b, 4d). Certainly the close adherence of hydrophobic polymer clusters [51] within the interior structure of the drug sphere may have influenced this process. Increased drug release with longer ultrasonication time (10-30 s) was associated with cracks and central sphere damage (Figure 4d). Oscillation, high fluid shear forces and heat generated by cavitation during ultrasonication $[46,52]$ are potential causes [50], and accelerating the dissociation of chlorhexidine from the compounds. Further work is needed to asses any potential mechanical property changes to the resin composites suggested in the literature $[42,53]$, or via ultrasonic treatment. It is also possible to reduce spheres size using temperature control [25] or nucleation catalysts and tune the ultrasonic treatments to optimise these outcomes.

Incorporation of novel chlorhexidine spheres with high drug content (90-95\%) in UDMAHEMA resins and the use of ultrasound demonstrated a responsive and lower chlorhexidine release, compared to chlorhexidine diacetate over $650 \mathrm{~h}$ water storage. Inclusion of the chlorhexidine spheres in a range of dental prostheses, via temporary soft lining materials and to treat denture stomatitis and a responsive ultrasonic drug release in maxillo-facial prostheses are realistic applications. The current results illustrate that these spherical chlorhexidine spheres may be beneficial in developing antibacterial dental composites and drug delivery systems to treat Periodontitis and Peri-implantitis. The authors have established the lack of cytotoxicity to fibroblasts and antimicrobial effectiveness of the chlorhexidine spheres, against 
a range of bacteria associated with these diseases and at a safer concentration than in current products [54]. The application of restorative materials to the supra-bony parts of the exposed implant surfaces in patients with Peri-implantitis led to no further bone loss, stability and noninflamed soft tissues in the short term [55]. The current technology might be useful in these low stress bearing applications, with drug release enhanced through ultrasound application. This could provide potential benefits in clinical situations where more drug is required on demand, to safely treat relatively severe or persistent infections and with less risk of cytotoxicity. This could be extremely desirable in Medicine and Dentistry in the safe treatment of recurrent infections or carries.

\section{Acknowledgements}

The authors thank Mr. Russell Bailey of Nanovision (QMUL) for help with SEM, Dr Dongsheng Wu for assistance in Confocal laser scanning microscopy. Dong Luo thanks for the financial support from the China Scholarship Council during his $\mathrm{PhD}$ study. 


\section{References}

[1] Moszner N, Salz U. New developments of polymeric dental composites. Prog. Polym Sci. 2001;26: 535-576.

[2] Ferracane JL. Resin composite--state of the art. Dent. Mater. 2011;27:29-38.

[3] Ferracane JL. Current Trends in Dental Composites. Crit Rev Oral Biol M. 1995;6:302-318.

[4] Kleverlaan CJ, Feilzer AJ. Polymerization shrinkage and contraction stress of dental resin composites. Dent. Mater. 2005;21:1150-1157.

[5] Ensaff H, O'Doherty DM, Jacobsen PH. Polymerization shrinkage of dental composite resins. J Eng. Med. 2001;215:367-75.

[6] Brunthaler A, Konig F, Lucas T, Sperr W, Schedle A. Longevity of direct resin composite restorations in posterior teeth. Clin Oral Invest. 2003;7:63-70.

[7] Zeng P, Rao A, Wiedmann TS, Bowles W. Solubility properties of chlorhexidine salts. Drug Dev Ind Pharm. 2009;35:172-176.

[8] Zeng P, Zhang G, Rao A, Bowles W, Wiedmann TS. Concentration dependent aggregation properties of chlorhexidine salts. Int. J. Pharm. 2009;367:73-78.

[9] Stanislawczuk R, Pereira F, Munoz MA, Luque I, Farago PV, Reis A, et al. Effects of chlorhexidine-containing adhesives on the durability of resin-dentine interfaces. J Dent. $2014 ; 42: 39-47$.

[10] Bertolini MM, Portela MB, Curvelo JA, Soares RM, Lourenco EJ, Telles DM. Resinsbased denture soft lining materials modified by chlorhexidine salt incorporation: an in vitro analysis of antifungal activity, drug release and hardness. Dent. Mater. 2014;30:793-798.

[11] Lim BS, Cheng Y, Lee SP, Ahn SJ. Chlorhexidine release from orthodontic adhesives after topical chlorhexidine treatment. Eur J Oral Sci. 2013;121:211-217. 
[12] Carrilho MRO, Carvalho RM, de Goes MF, di Hipólito V, Geraldeli S, Tay FR, et al. Chlorhexidine Preserves Dentin Bond in vitro. J Dent Res. 2007;86:90-94.

[13] Zhang JF, Wu R, Fan Y, Liao S, Wang Y, Wen ZT, et al. Antibacterial dental composites with chlorhexidine and mesoporous silica. J Dent Res. 2014;93:1283-1289.

[14] Shen C, Zhang NZ, Anusavice KJ. Fluoride and chlorhexidine release from filled resins. J Dent Res. 2010;89:1002-1006.

[15] Yan S, Cai X, Yan W, Dai X, Wu H. Continuous Wave Ultrasound Enhances Vancomycin Release and Antimicrobial Efficacy of Antibiotic-Loaded Acrylic Bone Cement In Vitro and In Vivo. J Biomed Mater Res B. 2007;82B:57-64.

[16] Huynh TT, Padois K, Sonvico F, Rossi A, Zani F, Pirot F, et al. Characterization of a polyurethane-based controlled release system for local delivery of chlorhexidine diacetate. Eur J Pharm Biopharm. 2010;74:255-264.

[17] Barbour ME, Maddocks SE, Wood NJ, Collins AM. Synthesis, characterization, and efficacy of antimicrobial chlorhexidine hexametaphosphate nanoparticles for applications in biomedical materials and consumer products. Int. J Nanomed. 2013;8:3507-3519.

[18] Hook ER, Owen OJ, Bellis CA, Holder JA, O'Sullivan DJ, Barbour ME. Development of a novel antimicrobial-releasing glass ionomer cement functionalized with chlorhexidine hexametaphosphate nanoparticles. J Nanobiotechnol. 2014;12:3.

[19] Badea M, Olar R, Iliş M, Georgescu R, Călinescu M. Synthesis, characterization, and thermal decomposition of new copper (II) complex compounds with chlorhexidine. J Therm Anal Calorim. 2012;111:1763-1770.

[20] Cheng L, Weir MD, Xu HH, Kraigsley AM, Lin NJ, Lin-Gibson S, et al. Antibacterial and physical properties of calcium-phosphate and calcium-fluoride nanocomposites with chlorhexidine. Dent. Mater. 2012;28:573-583.

[21] Kim H-J, Kwon T-Y, Kim K-H, Kwon S-T, Cho D-H, Son JS. Long-term release of 
chlorhexidine from dental adhesive resin system using human serum albumin nanoparticles. Polym Bull. 2014;71:875-586.

[22] Hendriks JGE, Ensing GT, van Horn JR, Lubbers J, van der Mei HC, Busscher HJ. Increased release of gentamicin from acrylic bone cements under influence of low-frequency ultrasound. J Control Release. 2003;92:369-374.

[23] Shrestha A, Fong SW, Khoo BC, Kishen A. Delivery of antibacterial nanoparticles into dentinal tubules using high-intensity focused ultrasound. J Endodont. 2009;35:1028-1033.

[24] Kishen A, Khoo BC, Shrestha A, Ohl SW. Characterizing bubble dynamics created by high-intensity focused ultrasound for the delivery of antibacterial nanoparticles into a dental hard tissue. Proc Inst Mech Eng H. 2010;224:1285-1296.

[25] Luo D, Shahid S, Wilson RM, Cattell MJ, Sukhorukov GB. Novel Formulation of Chlorhexidine Spheres and Sustained Release with Multilayered Encapsulation. ACS Appl Mater Inter. 2016;8:12652-12660.

[26] Tabary N, Chai F, Blanchemain N, Neut C, Pauchet L, Bertini S, et al. A chlorhexidineloaded biodegradable cellulosic device for periodontal pockets treatment. Acta Biomater. 2014;10:318-329.

[27] Yue IC, Poff J, Cortes ME, Sinisterra RD, Faris CB, Hildgen P, et al. A novel polymeric chlorhexidine delivery device for the treatment of periodontal disease. Biomaterials. 2004;25:3743-3750.

[28] Călinescu M, Negreanu-Pîrjol T, Georgescu R, Călinescu O. Synthesis and characterization of new copper(II) complex compounds with chlorhexidine. Part I. Cent Eur J Chem. 2010;8:543-549.

[29] Wu Y, Zhou N, Li W, Gu H, Fan Y, Yuan J. Long-term and controlled release of chlorhexidine-copper(II) from organically modified montmorillonite (OMMT) nanocomposites. Mater Sci Eng C. 2013;33:752-757. 
[30] Fischer HC, Chan WC. Nanotoxicity: the growing need for in vivo study. Curr Opin Biotech. 2007;18:565-571.

[31] Verraedt E, Pendela M, Adams E, Hoogmartens J, Martens JA. Controlled release of chlorhexidine from amorphous microporous silica. J Control Release. 2010;142:47-52.

[32] Duncan B, Li X, Landis RF, Kim ST, Gupta A, Wang LS, et al. Nanoparticle-Stabilized Capsules for the Treatment of Bacterial Biofilms. ACS Nano. 2015;9:7775-7782.

[33] Giunchedi P, Juliano C, Gavini E, Cossu M, Sorrenti M. Formulation and in vivo evaluation of chlorhexidine buccal tablets prepared using drug-loaded chitosan microspheres. Eur J Pharm Biopharm. 2002;53:233-239.

[34] Kashi TS, Eskandarion S, Esfandyari-Manesh M, Marashi SM, Samadi N, Fatemi SM, et al. Improved drug loading and antibacterial activity of minocycline-loaded PLGA nanoparticles prepared by solid/oil/water ion pairing method. Int. J Nanomed. 2012;7:221-234.

[35] Inagaki LT, Dainezi VB, Alonso RC, Paula AB, Garcia-Godoy F, Puppin-Rontani RM, et al. Evaluation of sorption/solubility, softening, flexural strength and elastic modulus of experimental resin blends with chlorhexidine. J Dent. 2016;49:40-45.

[36] Nerurkar MJ, Zentner GM, Rytting JH. Effect of chloride on the release of chlorhexidine salts from methyl methacrylate: 2-hydroxyethyl methacrylate copolymer reservoir devices. J Control Release. 1995;33:357-363.

[37] Wilson SJ, Wilson HJ. The release of chlorhexidine from modified dental acrylic resin. J Oral Rehabil. 1993;20:311-319.

[38] Hiraishi N, Yiu CK, King NM, Tay FR, Pashley DH. Chlorhexidine release and water sorption characteristics of chlorhexidine-incorporated hydrophobic/hydrophilic resins. Dent. Mater. 2008;24:1391-1399.

[39] Leung D, Spratt DA, Pratten J, Gulabivala K, Mordan NJ, Young AM. Chlorhexidinereleasing methacrylate dental composite materials. Biomaterials. 2005;26:7145-7153. 
[40] P.D. Riggs MB, M. Patel. Chlorhexidine release from room temperature polymerising methacrylate systems. Biomaterials. 2000;21:345-351.

[41] Huang X, Brazel CS. On the importance and mechanisms of burst release in matrixcontrolled drug delivery systems. J Control Release. 2001;73:121-136.

[42] Pallan S, Furtado Araujo MV, Cilli R, Prakki A. Mechanical properties and characteristics of developmental copolymers incorporating catechin or chlorhexidine. Dent. Mater. 2012;28:687-694.

[43] Lavon I, Kost J. Ultrasound and transdermal drug delivery. Drug Discov Today. 2004;9:670-676.

[44] Collis J, Manasseh R, Liovic P, Tho P, Ooi A, Petkovic-Duran K, et al. Cavitation microstreaming and stress fields created by microbubbles. Ultrasonics. 2010;50:273-279.

[45] Charasseangpaisarn T, Wiwatwarrapan C. The effect of various frequencies of ultrasonic cleaner in reducing residual monomer in acrylic resin. Ultrasonics. 2015;63:163-167.

[46] Joshi A, Raje J. Sonicated transdermal drug transport. J Control Release. 2002;83:13-22.

[47] Madarati AA, Qualtrough AJ, Watts DC. Factors affecting temperature rise on the external root surface during ultrasonic retrieval of intracanal separated files. J Endodont. 2008;34:10891092.

[48] Yiu CK, King NM, Carrilho MR, Sauro S, Rueggeberg FA, Prati C, et al. Effect of resin hydrophilicity and temperature on water sorption of dental adhesive resins. Biomaterials. 2006;27:1695-1703.

[49] Pearson GJ. Long term water sorption and solubility of composite filling materials. J Dent. 1979;7:64-68.

[50] Pitt WG, Husseini GA, Staples BJ. Ultrasonic Drug Delivery - A General Review. Expert Opin Drug Deliv 2004;1:37-56. 
[51] Hiraishi N, Yiu CK, King NM, Tay FR. Chlorhexidine release and antibacterial properties of chlorhexidine-incorporated polymethyl methacrylate-based resin cement. J Biomed Mater Res B. 2010;94:134-140.

[52] Husseini GA, Pitt WG. Micelles and nanoparticles for ultrasonic drug and gene delivery. Adv Drug Deliv Rev. 2008;60:1137-1152.

[53] Jedrychowski JR, Caputo AA, Kerper S. Antibacterial and mechanical properties of restorative materials combined with chlorhexidines. J Oral Rehabil. 1983;10:373-381.

[54] Chlorhexidine crystal forms and uses thereof in medicine. International (PCT) Patent Application No. PCT/GB2017/050752.

[55] Shahdad S, Rawlinson S, Gupta P, Patel M, Whiley R, Gamble E, Cattell M. A novel approach to treat Peri-implantitis using a resin-ionomer restorative material. J Dent Res. 2014;93, Special issue B: 640 . 


\section{List of Tables}

Table 1, Change in slope (SD) as a function of ultrasonication treatment.

\section{Table 1}

\begin{tabular}{lccc}
\hline Measured slope range (SD) & 0h-3h & $205 \mathrm{~h}-275 \mathrm{~h}$ & $425 \mathrm{~h}-500 \mathrm{~h}$ \\
\hline Resin with CDP * & $1.93(0.48)$ & $0.45(0.028)$ & $0.13(0.03)$ \\
Resin with CDP/ultrasonics & $2.18(0.63)$ & $4.40(0.42)$ & $1.31(0.55)$ \\
Resin with SCP* & $0.21(0.06)$ & $0.11(0.02)$ & $0.09(0.06)$ \\
Resin with SCP/ultrasonics & $0.22(0.01)$ & $1.09(0.54)$ & $0.87(0.11)$ \\
\hline & $*=$ No Ultrasonics
\end{tabular}




\section{List of Figures}

Figure 1, SEM images showing the particle distribution of: (a) chlorhexidine diacetate crystals (CDP); (b) spherical chlorhexidine particles (SCP), and (c, d) individual particle morphology at high magnification ( $c=S C P, d=C D P$ ); Crosssectional images of the UDMA-HEMA resin discs containing (e) chlorhexidine diacetate crystal and (f) spherical chlorhexidine particles.

Figure 2, TGA of the spherical chlorhexidine particles, chlorhexidine diacetate and calcium chloride.

Figure 3, Release kinetics of the UDMA-HEMA resin discs containing; (a) chlorhexidine diacetate and (b) chlorhexidine spheres after repetitive ultrasound treatment at; $0 \mathrm{~s}$ (black), $10 \mathrm{~s}$ (red), $20 \mathrm{~s}$ (blue) and $30 \mathrm{~s}$ (green) and terminated at the 14.3 $\mathrm{h}^{1 / 2}$. Resin discs containing; (c) chlorhexidine diacetate and (d) chlorhexidine spheres after repetitive ultrasound treatment for 30s at the start (red), 30s at start and $14.3 \mathrm{~h}^{1 / 2}$ (blue) and $30 \mathrm{~s}$ start, $14.3 \mathrm{~h}^{1 / 2}$ and $20.6 \mathrm{~h}^{1 / 2}$ (green).

Figure 4, Confocal images of UDMA- HEMA resin specimen containing rhodamine B labelled spherical chlorhexidine particles; (a) before and (b) after $650 \mathrm{~h}$ water storage; (c) SEM photomicrograph (and inset) of chlorhexidine diacetate resin disc cross-section after $650 \mathrm{~h}$ water storage and repetitive ultrasound treatment for $10 \mathrm{~s}$; (d) SEM photomicrograph (and inset) of chlorhexidine particle resin disc cross-section after $650 \mathrm{~h}$ water storage and repetitive ultrasound treatment for $10 \mathrm{~s}$. 
Figure S1: Calibration curve of chlorhexidine absorption peak versus concentration. 
Figure 1
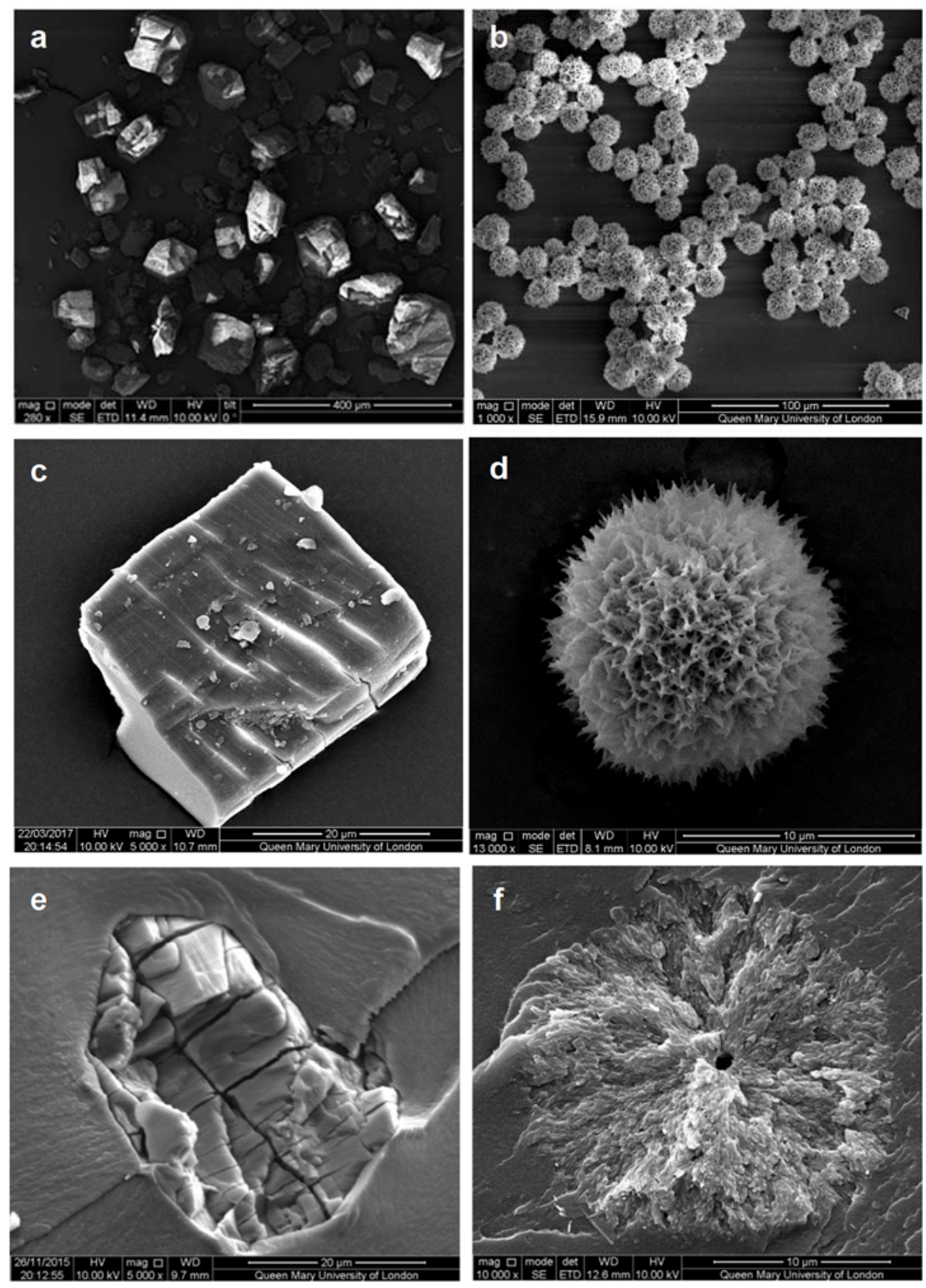
Figure 2

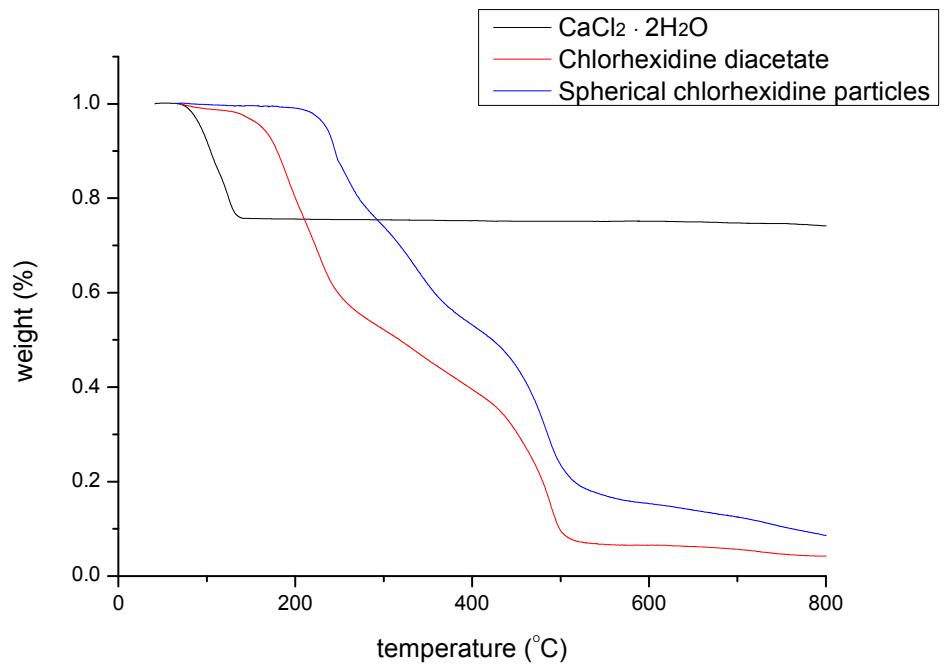


Figure 3
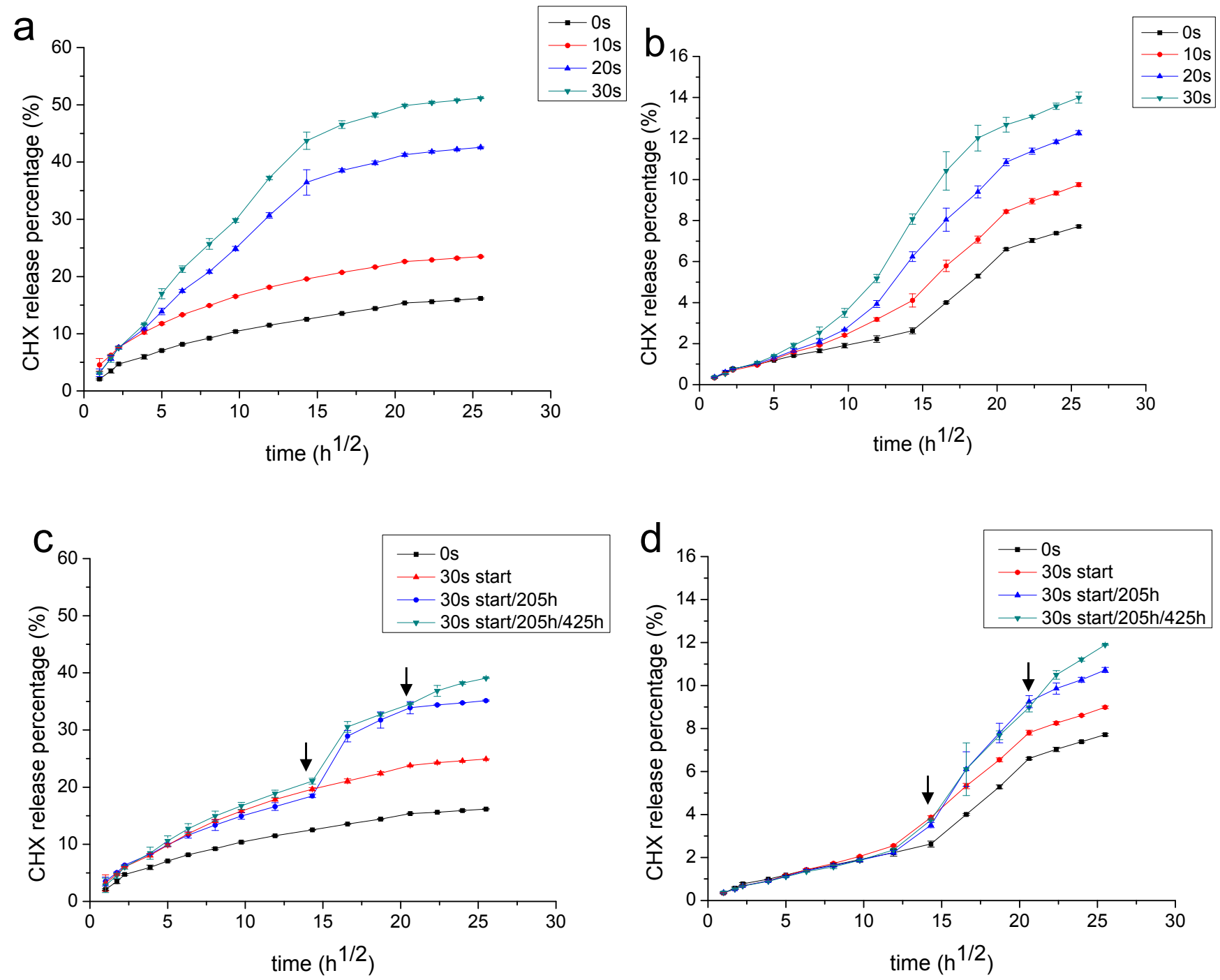

Arrows indicate ultrasonication time $\left(\mathrm{h}^{1 / 2}\right)$ 
Figure 4
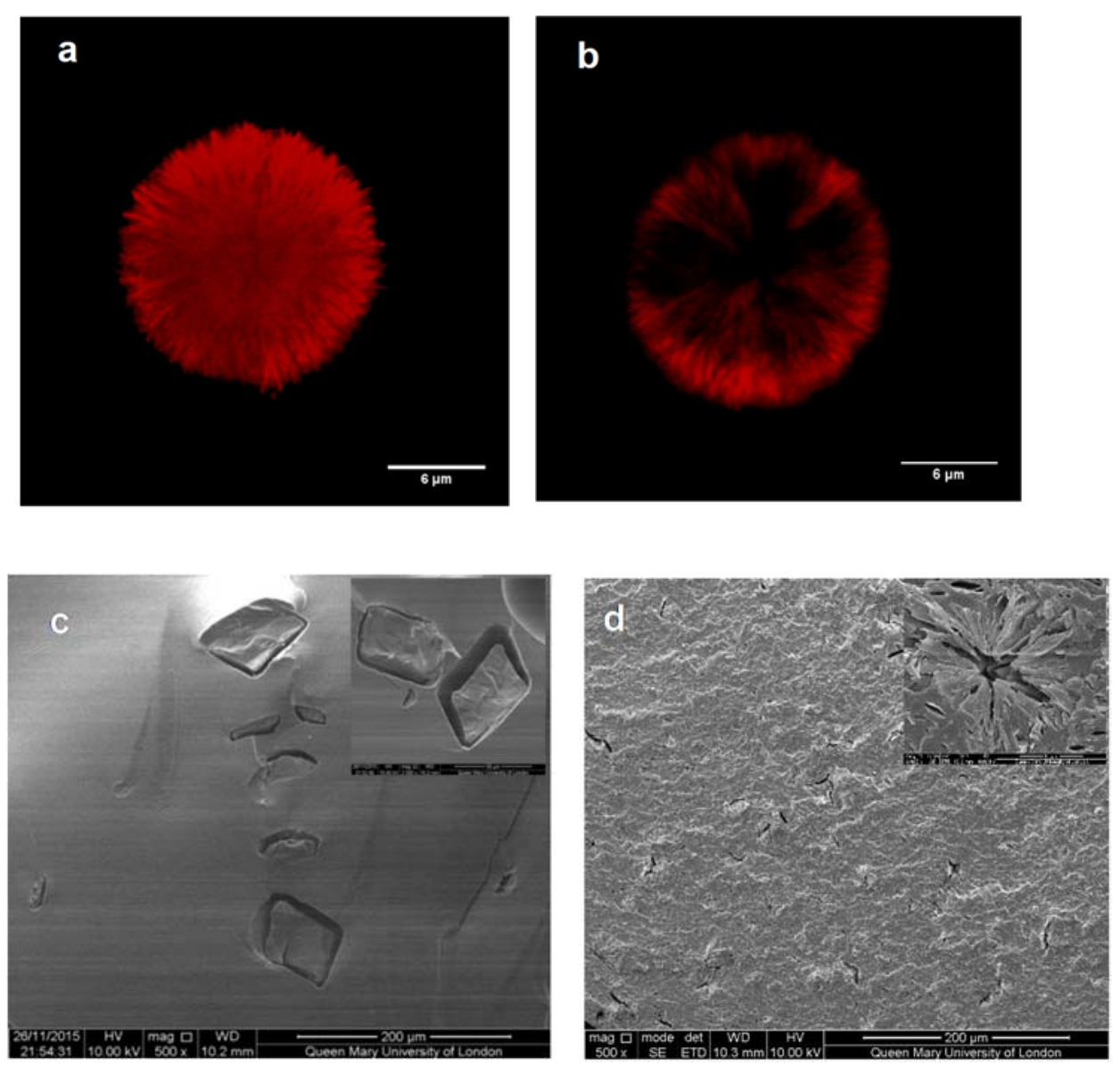


\section{Supplemental Information}

Figure S1

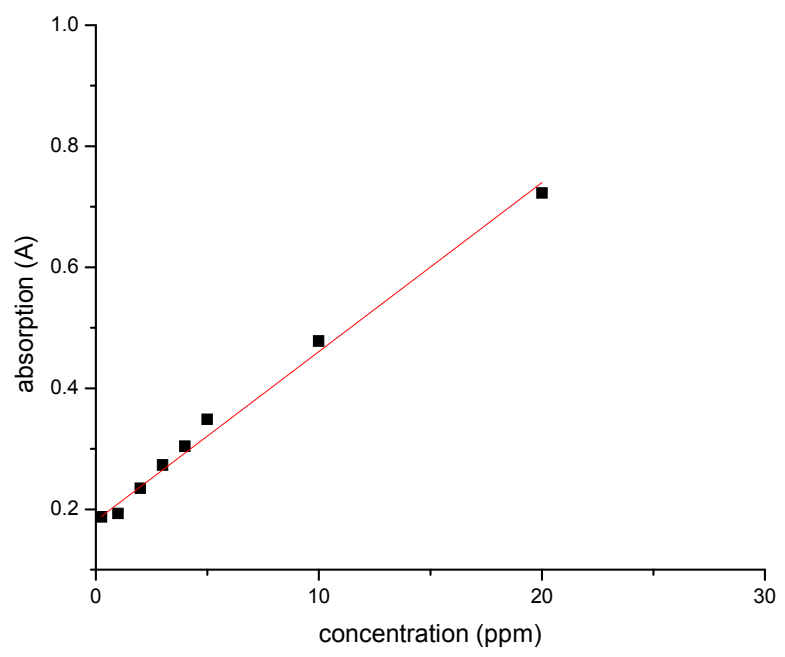

